\title{
BoS2
}

\section{SAGEEP2 Assessing Water Storage Changes on the Field Scale Combining Superconducting Gravimeter Observations with a Hydro-logical Model}

\author{
B. Creutzfeldt* (GFZ German Research Centre for Geoscience), A. Güntner \\ (GFZ German Research Centre for Geoscience), H. Wziontek (GFZ German \\ Research Centre for Geoscience) \& B. Merz (GFZ German Research Centre \\ for Geoscience)
}

\section{SUMMARY}

Information on water storages is crucial for many different applications, like agricultural production, groundwater recharge or transport of contaminants. Limitations of observation techniques and high spatiotemporal variability make the estimation of water storage challenging, especially for deeper zones.

Temporal gravimeter observations are significantly influenced by water storage changes (WSC) at the field scale and hence may provide valuable information about the state of the hydrological system.

In this study, we assess the benefit of temporal gravimeter measurements as an integral signal for hydrological application by evaluating a hydrological model using residuals time series of a superconducting gravimeter (SG). A simple conceptual model is used to estimate local WSC in the snow, soil, unsaturated saprolite, and saturated aquifer storage. The model is calibrated and evaluated against SG data on the one hand and several groundwater and/or soil moisture data on the other. The model is validated against independently estimated WSC derived from a state-of-the-art lysimeter.

The results show that using an SG as calibration constraint improves the model results substantially in terms of predictive capability and variation of the behavioral model runs in comparison to classical hydrological point measurements. Gravity measurements integrate over different hydrological storage components and the sampling volume is several orders of magnitude larger than that for the point measurements. The general problem of specifying the internal model structure or individual parameter sets can, however, not be solved with gravimeters alone. Additionally, the results show that also WSC in the deep vadose zone contribute significantly to the hydrological cycle, so SG might provide a tool to continuously and non-invasively monitor WSC also in this zone. 
Information on water storages is crucial for many different applications, like agricultural production, groundwater recharge or transport of contaminants. Limitations of observation techniques and high spatio-temporal variability make the estimation of water storage challenging, especially for deeper zones. Temporal gravimeter observations are significantly influenced by water storage changes (WSC) at the field scale and hence may provide valuable information about the state of the hydrological system.

In this study, we assess the benefit of temporal gravimeter measurements as an integral signal for hydrological application by evaluating a hydrological model using residuals time series of a superconducting gravimeter (SG). A simple conceptual model is used to estimate local WSC in the snow, soil, unsaturated saprolite, and saturated aquifer storage. The model is calibrated and evaluated against SG data on the one hand and several groundwater and/or soil moisture data on the other. The model is validated against independently estimated WSC derived from a state-of-the-art lysimeter.

The results show that using an SG as calibration constraint improves the model results substantially in terms of predictive capability and variation of the behavioral model runs in comparison to classical hydrological point measurements. Gravity measurements integrate over different hydrological storage components and the sampling volume is several orders of magnitude larger than that for the point measurements. The general problem of specifying the internal model structure or individual parameter sets can, however, not be solved with gravimeters alone. Additionally, the results show that also WSC in the deep vadose zone contribute significantly to the hydrological cycle, so SG might provide a tool to continuously and non-invasively monitor WSC also in this zone. 